\title{
Eiskeimbildung durch dielektrische Polarisation
}

\author{
Von Walter RaU \\ Aus der Forschungsstelle für Physik der Stratosphäre in der Max-Planck-Gesellschaft, Weißenau \\ (Z. Naturforschg. 6 a, 649-657 [1951]; eingegangen am 11. Juli 1951)
}

Herrn Professor E rich Regener zum 70. Gebutstag

In unterkühlten Wassertropfen kann die Eiskeimbildung durch ein elektrisches Feld in streng reproduzierbarer Weise hervorgerufen werden. Passende Elektrodenanordnungen (Funke im Luftraum auf die Tropfen bzw. Spitzenwirkung, Elektroden mit Bernsteinhülle, Kondensatoren und Funken in den Wassertropfen selbst) lassen erkennen, daß dabei die dielektrische Polarisation im unterkühlten Wasser wirksam ist. Gefrierkerne und Erschütterungen konnten ausgeschlossen werden.

Diese Eisbildung gelingt schon ab $-1^{\circ} \mathrm{C}$. Die benötigte Feldstärke hängt von der Unterkühlungstemperatur und vom Reinheitsgrad des Wassers ab; geringe Unterkühlungen erfordern höhere Feldstärken. Charakteristisch ist, daß immer sehr viele getrennte Eisteilchen (Sterne und Kreisscheibchen) gleichzeitig in einem Tropfen entstehen, und daß diese gegebenenfalls so orientiert sind, daß sie mit einer Diagonalen (Nebenachse) in Richtung der Feldlinien stehen.

Der Abtrocknungseffekt der Gefrierkerne und die Impfung von unterkühlten Tropfen mit trockenen Kernen (erstes Maximum des Gefrierkernspektrums) können somit durch die mit der Entstehung einer Doppelschicht verbundene dielektrische Polarisation erklärt werden.

Diese elektrische Eisbildung kann bei hohen Feldstärken auch in der Atmosphäre vorkommen; Gefrierkerne sind dann überflüssig. Auch beim Zerblasen (Lenard-Effekt) von Wassertropfen kann es damit zur Eisbildung kommen, sobald es sich um unterkühlte Wassertropfen handelt.

$\mathrm{N}^{\mathrm{sit}}$ Noner mane bildung in der Atmosphäre sowie wahrscheinlich auch bei der Entstehung der Gewitterelektrizität. Dadurch hat das alte Problem der Unterkühlbarkeit des Wassers und die Frage nach den Ursachen des im unterkühlten Wasser plötzlich einsetzenden Kristallisationsvorganges wieder besondere Bedeutung •erlangt. Die Schwierigkeiten, die der experimentellen Klärung dieser Vorgänge entgegenstehen, rühren hauptsächlich davon her, daß die Einzelversuche auch bei noch so genauer Einhaltung gleicher Versuchsbedingungen durch unkontrollierbare Faktoren beeinflußt werden und zum Teil verschieden ausfallen. So gelang es nicht, von der Impfung mit Eiskristallen abgesehen, die ja nur einen Wachstumsvorgang und keine Phasenentstehung darstellt, die Eisbildung im unterkühlten Wasser wirklich reproduzierbar zu veranlassen und damit die wesentlichen Ursachen zu erkennen.

Zuerst hatte man geglaubt, daß Erschütterungen, auch leichtester Art, die Kristallisation auslösen würden. Diese Ansicht ist auch heute noch sehr verbreitet, obgleich schon von $\mathrm{Fahrenheit,} \mathrm{der} \mathrm{als}$ erster die Unterkühlbarkeit beobachtet hatte, bemerkt und inzwischen mehrfach bestätigt worden ist, daß Erschütterungen durchaus nicht in jedem Falle zur Kristallisation führen und nur bei bestimmten Versuchsanordnungen (z. B. offenen Gefäßen) wirksam sind. Daher wurde die Eiskeimbildung später immer mehr den fremdstofflichen Beimengungen, den sogenannten „Kernen“ zugeschrieben. Da sich die Anwesenheit von geeigneten Kernen in den Wasserproben mit Sicherheit weder nachweisen noch verneinen läßt und es auch praktisch unmöglich ist, solche feinen suspendierten Teilchen aus dem Wasser ganz zu entfernen, kann diese Hypothese dem wechselvollen Charakter der Versuchsergebnisse immer gerecht werden; aber die Aussagen über die spezifischen Eigenschaften der eisbildenden Kerne waren nicht frei von Widersprüchen.

Neue Beweise für die Richtigkeit der Kernhypothese sind durch unsere Beobachtungen an individuellen Gefrierkernen und Untersuchungen über das Spektrum der natürlichen Gefrierkerne erbracht worden ${ }^{1}$. Insbesondere gelang dabei die Gefrierauslö-

1 W. R a u, Schr. deutsch. Akad. Luftfahrtforschg. 8, 65 [1944]; W. R a u, Z. Naturforschg. 5 a, 667 [1950]. 
sung im unterkühlten Wasser in stets reproduzierbarer Weise mit allen (untersuchten) Substanzen, also auch solchen, die normalerweise in der Atmosphäre vorkommen. Die Struktur der Kerne war im untersuchten Temperaturbereich bis $-20^{\circ} \mathrm{C}$ ohne wesentlichen Einfluß. Die Substanzen mußten nur fein gepulvert und in möglichst trockenem Zustand in die unterkühlten Tropfen eindringen. Die Kerne werden nämlich beim Aufenthalt in feuchter Luft oder in Wasser allmählich inaktiv, durch Abtrocknen aber wieder besonders aktiv.

Zur Deutung dieser merkwürdigen Kernwirksamkeit (Abtrocknungseffekt), die wir auch bei der atmosphärischen Eisbildung nachweisen konnten ${ }^{2}$ und die inzwischen auch von anderer Seite an „künstlichen“ Gefrierkernen beobachtet worden ist ${ }^{3}$, hatten wir angenommen, daß die am trockenen Kern beim Eindringen in das Wasser immer entstehende elektrische Doppelschicht, vermöge ihrer ausrichtenden Wirkung auf die Wasserdipole, beim Eindringen in unterkühltes Wasser die Keimbildung einleitet. Dann müßte die Eisbildung auch durch ein elektrisches Feld allein hervorzurufen sein. Die vorliegende Arbeit erbringt die Bestätigung dieser Vermutung und den Nachweis, daß bei dieser neuen Art der Eiskeimbildung nur die dielektrische Polarisation zur Wirkung kommt, und daß das elektrische Feld nicht einfach indirekt über Gefrierkerne oder besondere, evtl. doch mögliche keimbildende Erschütterungen wirksam ist.

\section{Die Untersuchungsmethode}

Die Versuche wurden mit der früher schon beschriebenen Kältekammer an einzelnen unterkühlten Wassertropfen $\left(0,6\right.$ bis $\left.0,8 \mathrm{~cm}^{3}\right)$ ausgeführt, welche meist auf einer hochglanzpolierten Chromfläche lagen. Soweit eine isolierende Unterlage benötigt wurde, fanden Glas, Plexiglas oder Bernstein Verwendung. Das elektrische Feld wurde mittels verschieden gearteter Elektroden angelegt. Während der Kühlungen blieb die Kammer geschlossen. Die bei dieser Apparatur mögliche getrennte Kühlung von Kammerluft und Tropfenunterlage wurde immer so einreguliert, da $\beta$ der Tropfen dieselbe Temperatur wie die Kammerluft besaß. Die Temperaturmessung erfolgte mit Thermoelementen. Ein Ventilator sorgte wieder für gleichmäßige Temperaturverteilung in der Kammer.

Die Wassertropfen enthalten natürlich auch Gefrierkerne. Um deren Wirkung sicher von der elektrischen Gefrierauslösung trennen zu können, wurde vor und nach Kühlungen mit elektrischem Feld auch der gewöhnliche Gefriereinsatz in den Tropfen ohne Feld bestimmt und das Spektrum dieser Gefrierkerne ermittelt. Dieselben

2 W. R a u, Wetter Klima 1, 81 [1949].

3 L. F a c y, J. Sci. Météorologie 2, 13 [1950]; E. M. Fournier d'A l be, J. Glaciology 1, 311 [1949].
Tropfen wurden also mehrmals nacheinander zum Erstarren gebracht und wieder aufgetaut. Diese Kontrolle war um so notwendiger, als bei den Versuchen z. Tl. verschiedene Unterlagen für die Tropfen und auch in die Wassertropfen hineinragende Elektroden Verwendung fanden, was vielleicht einen Einfluß auf die Gefriertemperaturen hätte haben können. Ein genauer Überblick über die Effekte und die Reproduzierbarkeit der Einzelversuche konnte erst aus längeren Versuchsserien und den daraus ermittelten Häufigkeitsverteilungen der beobachteten $\mathrm{Ge}-$ friertemperaturen gewonnen werden.

\section{Versuchsergebnisse}

1. Ges a m tergebnis

Der deutlichste Beweis für die Wirkung des elekrischen Feldes ist dadurch gegeben, daß die Eisbildung sofort beim Anlegen der Hochspannung einsetzt, die Keimbildung also „eingeschaltet“ werden kann. Das ist ein ganz wesentlicher Unterschied gegenüber der Kristallisation an Gefrierkernen, die, von Impfung abgesehen, immer zeitlich ganz unvermittelt einsetzt und nicht beeinflußt werden kann. Die erzielte Wirkung kommt am besten in der Häufigkeitsverteilung aller in unseren Versuchen bei elektrischer Gefrierauslösung aufgetretenen Erstarrungstemperaturen zum Ausdruck (Abb. 1, Kurve 1). Diese Kurve gilt nur für gut destilliertes Wasser.

In Zwischenversuchen ohne elektrische Auslösung ergaben dieselben Wassertropfen die Häufigkeitsverteilung der Kurve 2, welche bei allerdings wesentlich geringerer Statistik wieder eine recht gute Bestätigung des früher mitgeteilten allgemeinen Gefrierkernspektrums (Kurve 3) darstellt, das bei der großen Anzahl Einzelversuche, die zur Ermittlung von Kurve 1 diente, auch dort hätte herauskommen müssen, wenn es sich um die Wirkung von Gefrierkernen gehandelt hätte ${ }^{4}$. Man sieht also, daß die Gefrierauslösung durch ein elektrisches Feld schon wenige Grade unter dem Nullpunkt erfolgen kann, wo ein „Kerngefrieren“ noch sehr selten ist. Das im gleichen Temperaturbereich liegende erste Maximum des Gefrierkernspektrums (Kurve 3) rührt von der absichtlichen Impfung mit trockenen Kernen her, die hier tunlichst vermieden wurde.

Von besonderer Bedeutung bei der elektrischen Eisbildung ist die Reinheit des Wassers. Schon in weniger sorgfältig destilliertem Wasser, in Leitungswasser oder absichtlich chemisch leicht verunreinig-

4 Wegen der geringen Abweichung vom allgemeinen Gefrierkernspektrum sei auf die ausführliche Diskussion in der zitierten Arbeit W. Rau, Z. Naturforschg. 5a, 667 [1950], Abschnitt V, verwiesen. 
tem Wasser gelingt die elektrische Eisbildung nicht mehr so gut (Abb. 1, Kurve 4), obgleich das Kerngefrieren in denselben Tropfen noch ganz normal ist (Kurve 5). Solches Wasser muß man merkwürdigerweise bis in den Temperaturbereich des zweiten Maximums des Gefrierkernspektrums unterkühlen, um das Gefrieren elektrisch auslösen zu können. Die

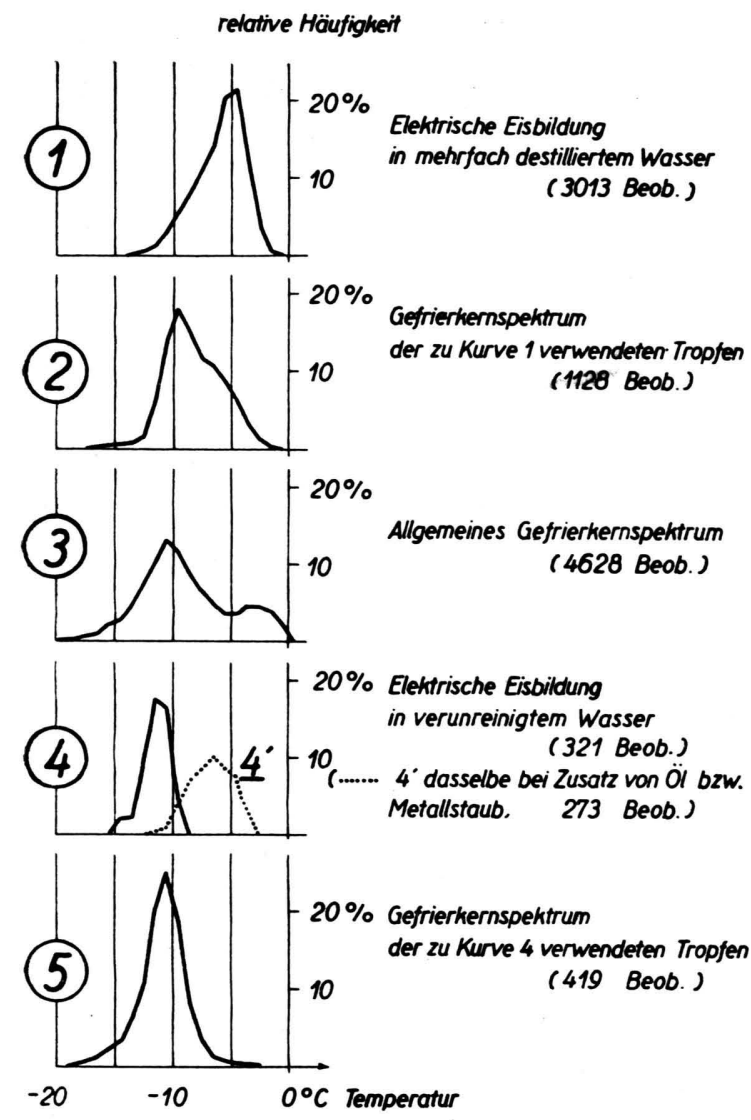

Abb. 1. Gefriertemperaturen bei elektrischer Auslösung und Gefrierkernspektren.

Unterscheidung von dem im gleichen Temperaturbereich sehr häufigen Gefrieren durch Kerne ist durch die schon erwähnte zeitliche Koinzidenz des Gefriereinsatzes mit dem Anlegen des elektrischen Feldes und durch die Eisstruktur möglich.

Die Wichtigkeit der Reinheit des Wassers ist hier verständlich, denn die elektrischen Eigenschaften des Wassers werden ja schon durch geringste Verunreinigungen stark geändert. Offenbar kann das elektrische Feld in reines Wasser eindringen und die für die Eiskeimbildung verantwortliche dielektrische Polarisation hervorrufen. Bei jeder Verunreinigung ist je- doch mit einer gewissen Leitfähigkeit zu rechnen, das Feld bricht schon in der Oberfläche zusammen, wird dadurch abgeschirmt und kann dann im Innern nicht mehr keimbildend wirken.

Daß gerade die Temperaturen bevorzugt sind, bei denen die beiden Maxima des Gefrierkernspektrums liegen, könnte vermuten lassen, daß die elektrische Gefrierauslösung nur eine besondere Art der Erzeugung günstiger Kerne wäre, besonders weil die oft behauptete sehr enge Bedingung der Isomorphie der Kerne mit Eis nach unseren Ergebnissen nicht eingehalten werden muß. Diese Vermutung konnte durch die folgenden Einzeluntersuchungen widerlegt werden.

\section{Einzeluntersuchungen}

a) Eiskeimbildung durch elektrische Funken

In der Doppelschicht, einem elektrischen Feld von molekularen Dimensionen, dessen Spannung, die „Berührungsspannung“, in der Größenordnung von 0,1 bis 1 Volt angenommen werden kann $^{5}$, muß man mit sehr hohen Feldstärken rechnen. Daher wurden die ersten Untersuchungen mit möglichst hohen Feldern ausgeführt.

Die Chromunterlage des Wassertropfens wurde mit dem negativen Pol der Hochspannungsquelle verbunden. Eine Messingkugel ( $5 \mathrm{~mm}$ Durchm.) als positive Elektrode mit Spannungen bis $7 \mathrm{kV}$ möglichst nahe über dem Tropfen, blieb unwirksam. Erst von einer Spitze (Platin, Messing, Kohle) ausgehende Funken gaben die erwartete Wirkung. Dabei wechselten Entladungsform und Eisbildung mit der Reinheit des Wassers. Bei reinem Wasser springen einzelne Gleitfunken von der positiven Spitze zur Tropfenoberfläche und daran entlang zur Metallunterlage über (Aufnahme $1^{*}$ ). Der Tropfen wird dadurch flach gedrückt; oft werden auch Teile desselben weggerissen und zerspritzt. Trotzdem kann die Eisbildung noch ausbleiben, ein Zeichen, daß auch sehr heftige Erschütterungen keine Eiskeimbildung bringen. Spätestens nach einigen Funkenüberschlägen setzt dann aber die Eisbildung ein, und wenn darauf der Tropfen wieder geschmolzen und erneut gekühlt wird, erfolgt die Eisbildung stets beim ersten Funkenübergang sofort. Die Temperatur muß dabei um -3 bis $-4^{\circ} \mathrm{C}$ liegen. Dieser Vorgang läßt sich mehrmals wiederholen. Meist werden

5 Grimseh l-Tomas chek, Lehrbuch der Physik II, 9. Auflage, S. 101

* Aufnahmen $1-20$, s. Tafel, S. 652 b, c u. d. 
die Gleitfunken allmählich seltener, die Ableitung in der Oberfläche wird stärker und die Unterkühlungstemperatur muß dann tiefer sein. Die Entladung nimmt immer mehr die Form einer kurzen Büschelentladung an (Aufnahme 2); der Tropfen bleibt beim Funkenüberschlag ganz ruhig. Dann ist die Eisbildung durch den elektrischen Funken erst im Gebiet um $-10^{\circ} \mathrm{C}$ möglich.

Der Wassertropfen ist durch den häufigen Funkenüberschlag verunreinigt worden. Das läßt sich an absichtlich verunreinigten Wassertropfen nachweisen. Schon ein Zusatz von etwa 10\% Leitungswasser zu in Jenaer Glasgeräten mehrmals destilliertem Wasser genügt, um die Gefrierauslösung vom ersten ins zweite Maximum zu verschieben. Auch in Metallapparaten destilliertes Wasser erwies sich in diesem Sinne schon als verunreinigt.

Besonders bemerkenswert ist, daß sich die Eisgebilde bei der durch Funkenüberschlag erzwungenen Keimbildung von dem gewöhnlichen an Gefrierkernen gewachsenen Eis wesentlich unterscheiden. Beim gewöhnlichen Gefrieren erstarrt der ganze Tropfen von einer einzigen Eiskeimstelle aus in zusammenhängenden Gebilden, wobei im Temperaturgebiet bis $-8^{\circ} \mathrm{C}$ in Übereinstimmung mit Schneebeobachtungen nur Eisnadeln vorkommen ${ }^{1}$, während erst darunter das flächenhafte Wachstum zu sechseckigen Plättchen und Sternen auftritt. Dagegen ist bei der Eiskeimbildung durch elektrische Funken meistens der ganze Tropfen schlagartig erfüllt von einer Unzahl von sechseckigen Sternplättchen und Kreisscheibchen (Aufnahme 3 und 4), und zwar schon bei Temperaturen um -3 bis $-4^{\circ} \mathrm{C}$. Diese Eissternchen werden oft in den Tropfen herumgewirbelt und schwimmen schließlich, glasklar durchsichtig, an der Tropfenoberfläche. Der Wassertropfen erstarrt dann ganz langsam, indem eine horizontal scharf begrenzte Eisschicht vom Tropfengrund, d. h. von der kühlenden Metallplatte, her in die Höhe wächst, wobei die kleinen Eiskristalle sogar wieder abschmelzen können. Auch dieses Eis ist so durchsichtig, daß es oft übersehen werden kann; es zeigt höchstens eine leichte Facettierung. Diese ist in dem hellen Fleck der Aufnahme 8 unten im reflektierten Licht zu erkennen. Bei tieferen Entstehungstemperaturen wachsen nur wenige größere, flächige Eisgebilde von der Auftreffstelle des Funkens aus rasch durch den

6 Die Aufnahme 5 zeigt an der durch Pfeile markierten Stelle das Spiegelbild der Elektrode: 0,2 mm Pt-Draht in einem dickeren Metallstäbchen sitzend. Das Eis wächst von der Auftreffstelle des Funkens, senkrecht unter der Spitze des Pt-Drahtes, radial aus.
Tropfen hindurch (Aufnahme 5); dieses Eis ist dann stark reflektierend weiß, wie es in diesem Temperaturbereich auch beim Kerngefrieren immer der Fall ist ${ }^{6}$.

Da die dielektrische Polarisation im wesentlichen eine Orientierung molekularer Dipole bedeutet, sollten die durch sie erzeugten Eisgebilde eine besondere Ausrichtung haben. Das ist in vielen Fällen tatsächlich zu beobachten. Die Sternchen oder runden Eisscheibchen stehen, oft mehrere unter sich, parallel in Ketten längs der Projektion der Funkenwege auf dem Tropfengrund, mit den Nebenachsen in der Feldlinienrichtung, die unterdrückte Hauptachse senkrecht dazu (Aufnahme 8 und 9). Ihr einige Zeit andauerndes Hin- und Herpendeln könnte von anhaftenden Ladungen herrühren. Die Anordnung der Eisplättchen erinnert an die bei Funkenüberschlägen auf Dielektrika auftretenden Lichtenberg-Figuren, deren Zustandekommen man sich so vorstellt, daß auf dem Funkenweg haftenbleibende Ladungen den aufgestreuten Staub anordnen; diese Markierung würde hier durch die längs des Funkenweges gebildeten Eiskeime (Aufnahme 6-8) erfolgen ${ }^{7}$.

Funken lösen das Gefrieren auch aus, wenn man Wassertropfen auf isolierende Unterlagen bringt und den Funken zwischen 2 Spitzen überschlagen läßt. Dabei genügt es nicht, daß der Funke irgendwo in der Nähe überspringt, auch nicht, wenn die Funkenstrecke so gestellt wird, daß verdampfendes Elektrodenmaterial bzw. Ionen, die möglicherweise als Gefrierkerne wirken könnten, in den Tropfen gelangen. Der Funke muß sich dem Tropfen richtig anlegen. Besonders eindrucksvoll ist die Wirkung, wenn man mehrere kleine Tröpfchen in die Funkenstrecke bringt und diese dann alle auf einen Schlag erstarren.

In diesem Zusammenhang wäre noch zu erwähnen, daß an Eistropfen immer nur die kurze Büschelentladung wie bei verunreinigtem Wasser (Aufnahme 2) auftritt, auch wenn sie im geschmolzenen Zustand wieder Gleitfunken ergeben. Ob darin das vom Wasser abweichende dielektrische Verhalten des Eises zum Ausdruck kommt, oder ob beim Gefrieren gewisse Beimengungen in der Oberflächenschicht angereichert werden und die andere Entladungsform bedingen, konnte bis jetzt nicht eindeutig entschieden werden. Daß im Temperaturgebiet um $-10^{\circ} \mathrm{C}$

7 In Aufnahme 6 ist wieder die Auftreffstelle des Funkens, durch Pfeile markiert, an dem dort entstandenen gekreuzten Kristall zu erkennen; von dieser Stelle aus verlaufen einzelne gebogene Äste zum Tropfenrand. Die Aufnahmen 7 und 8 zeigen solche Äste, aus einzelnen Eisplättchen bestehend, in 20-facher Vergrößerung. 


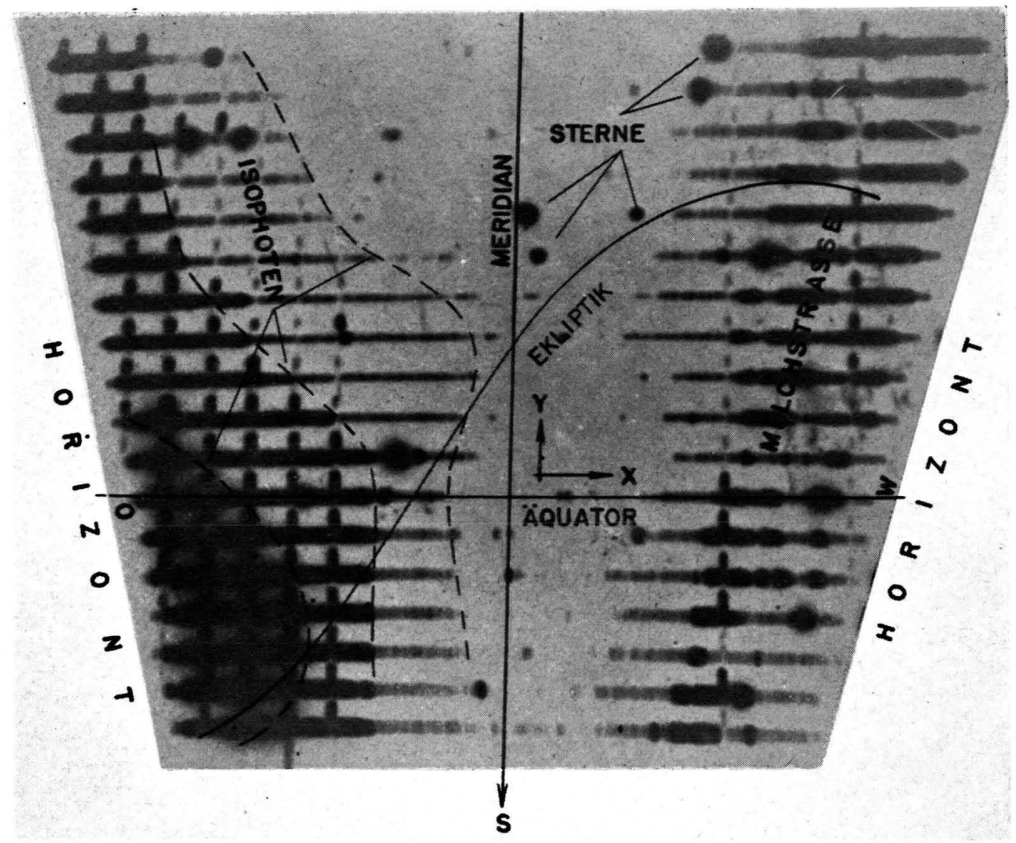

Abb. 1. Registrierung des Zodiakallichtes. Sternzeit $10^{\mathrm{h}} 24^{\mathrm{m}}, 9$. Dez. 1950. Das Zodiakallicht erscheint über dem östlichen Horizont. Die Registrierlinien sind um $3^{\circ}$ in der Deklination $y$ voneinander getrennt. Die Eichmarken für den Stundenwinkel $x$ sind über dem östlichen Horizont um $10^{\triangleleft}$ und über dem westlichen Horizont um $30^{\circ}$ voneinander getrennt. Der Maßstab ist ungefähr um einen Faktor 3 zugunsten der Deklination $y$ gegenüber dem Stundenwinkel $x$ vergrößert. 
W.Rau, Eiskeimbildung durch dielektrische Polarisation. (S. 649)
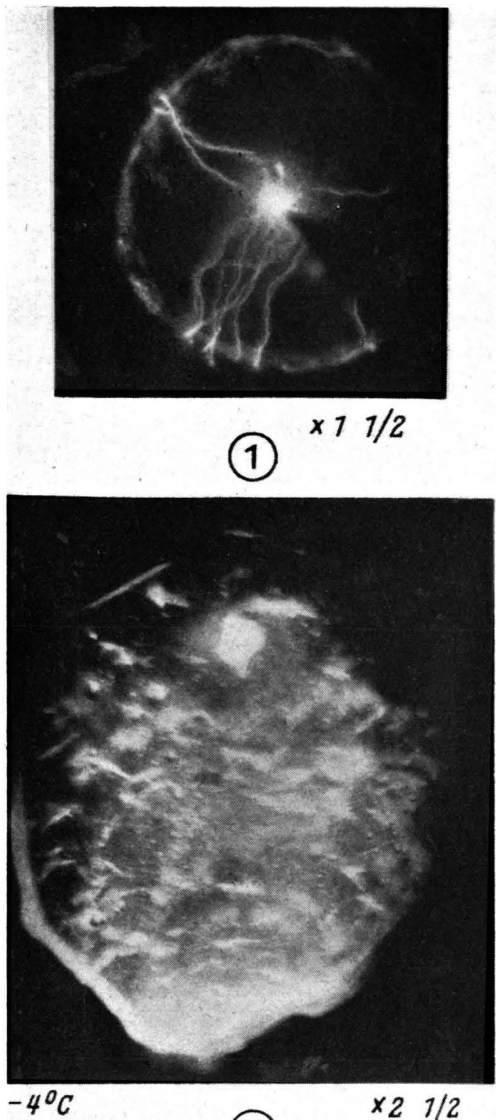

(3)

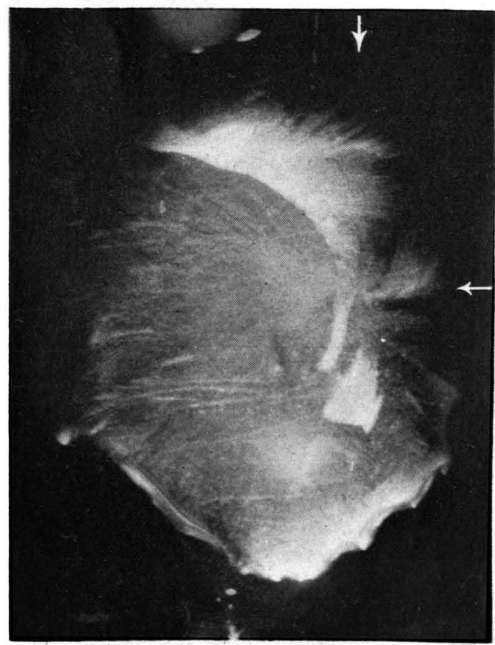

(6)

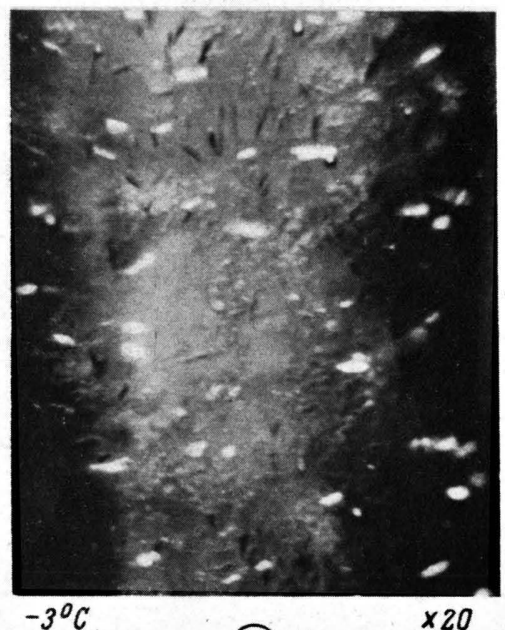

(4)

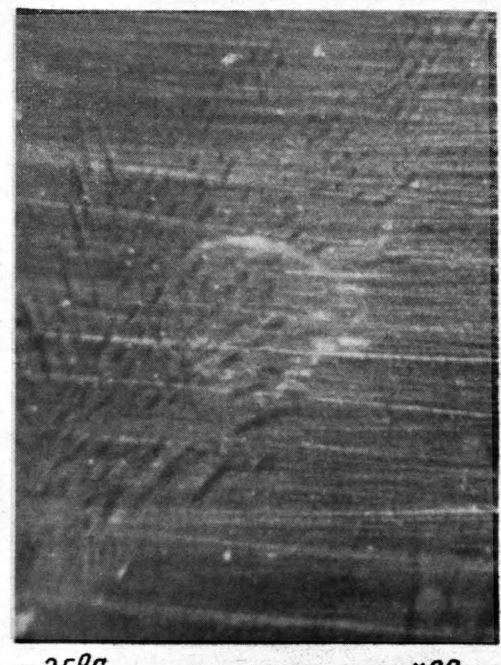

(7)

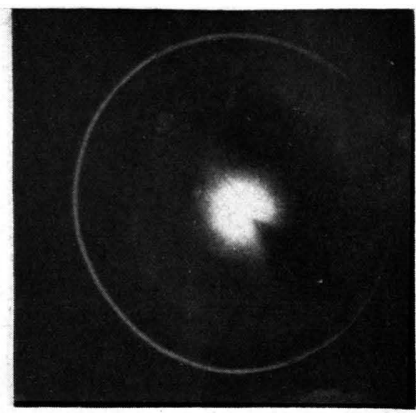

(2)

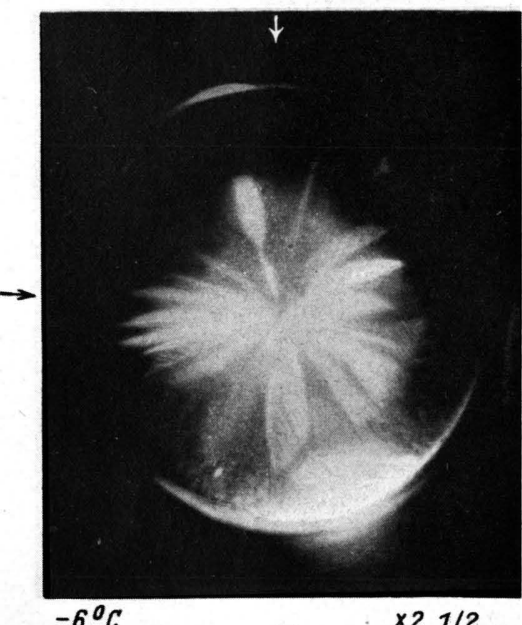

(5)

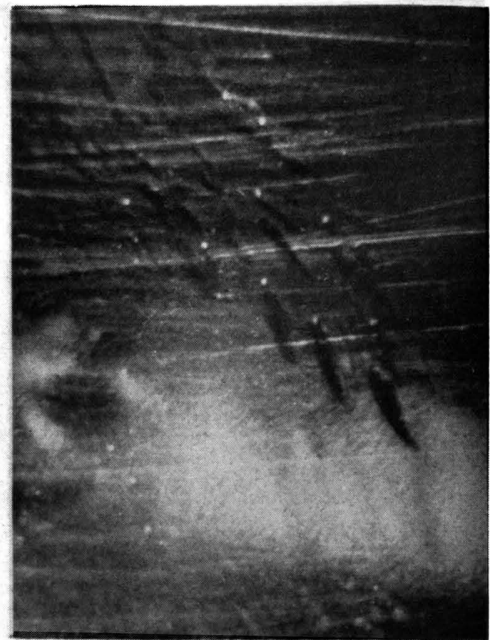

$-3,5^{\circ} \mathrm{C}$

(8)

Aufnahmen $1-8$ 

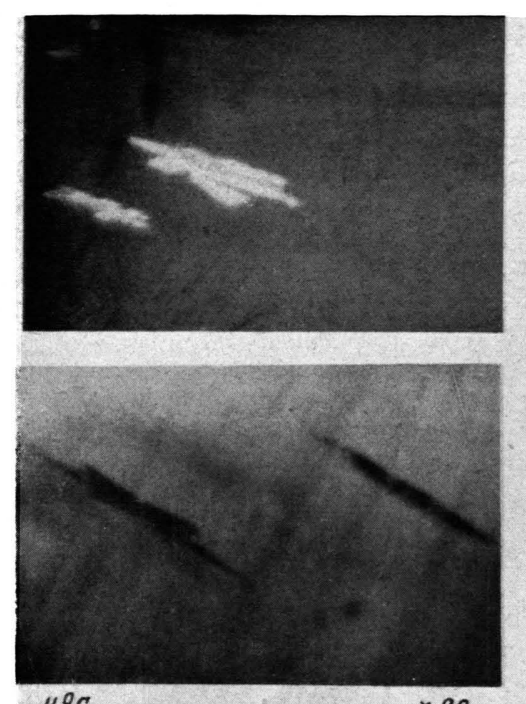

(9)

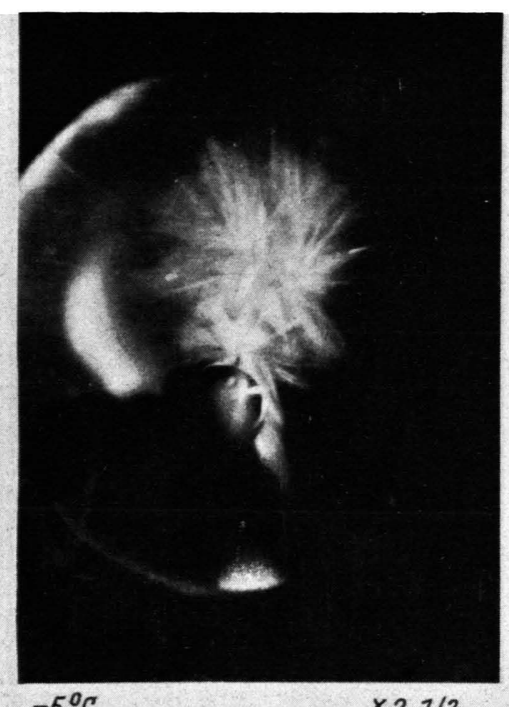

(10)

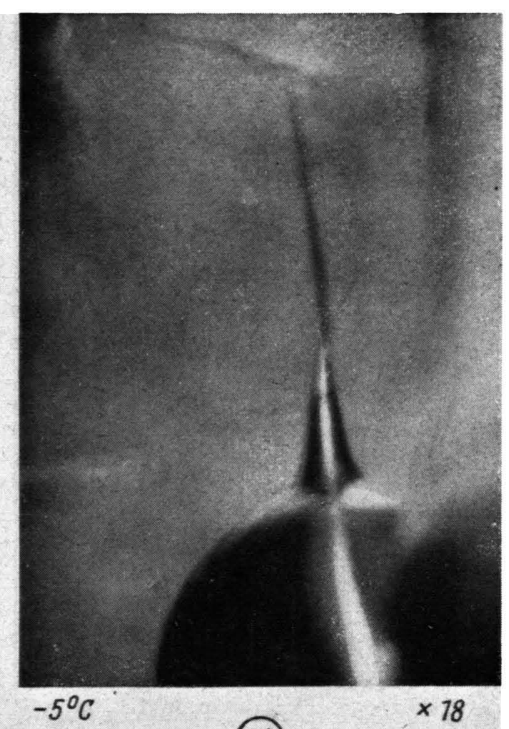

(11)

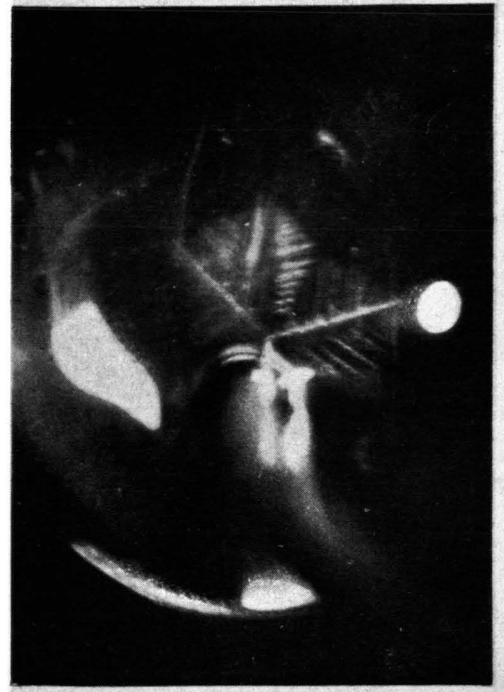

(12)

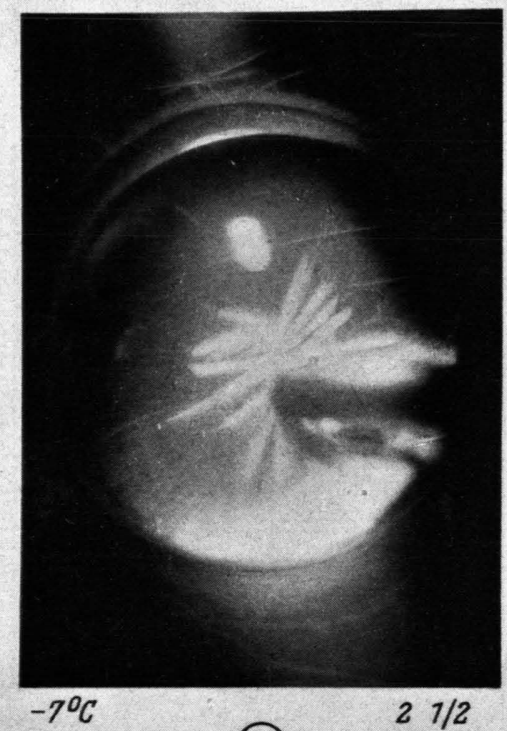

(13)

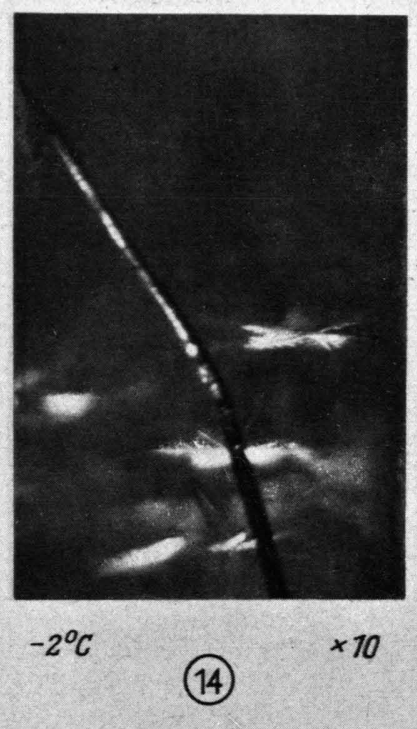

Aufnahmen 9-14. 


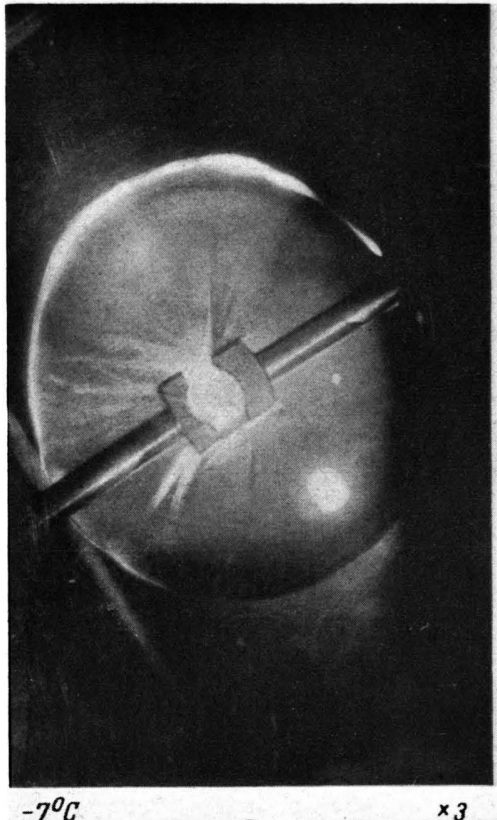

(15)

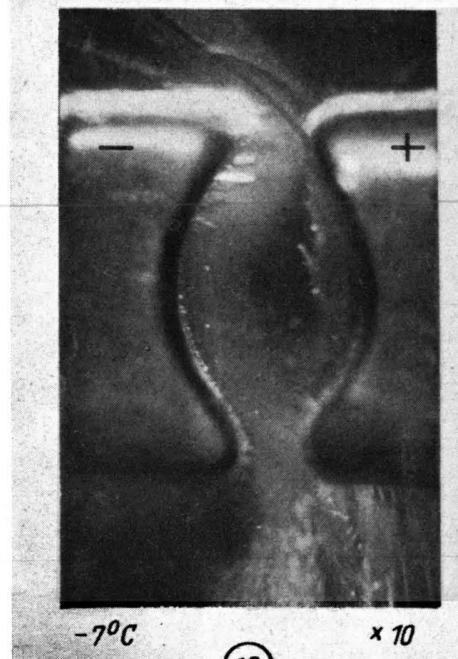

(18)

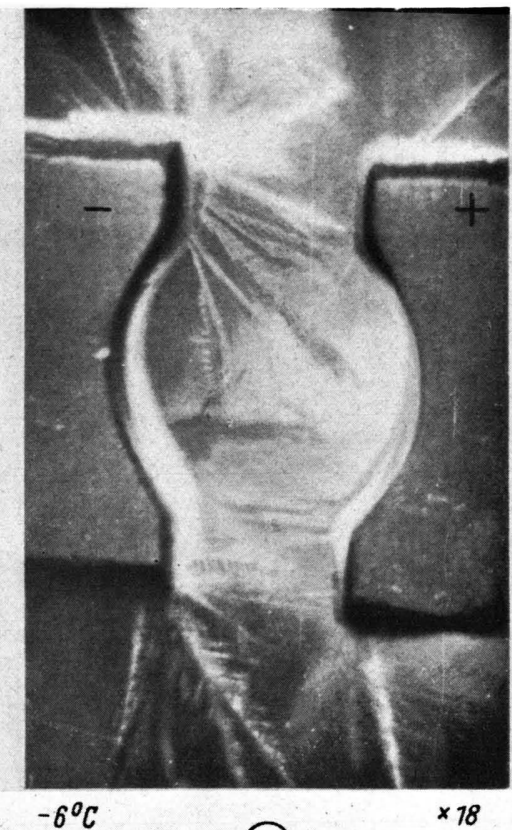

(16)

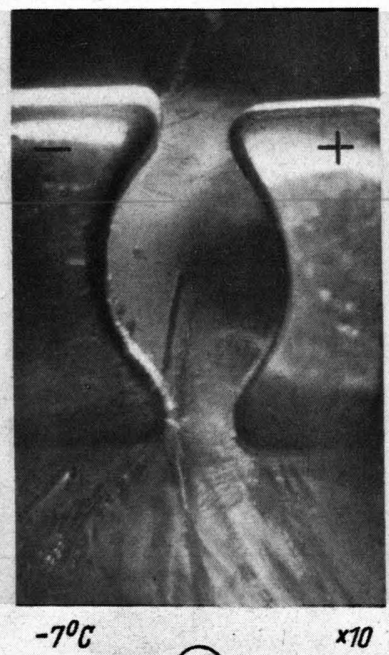

(19)

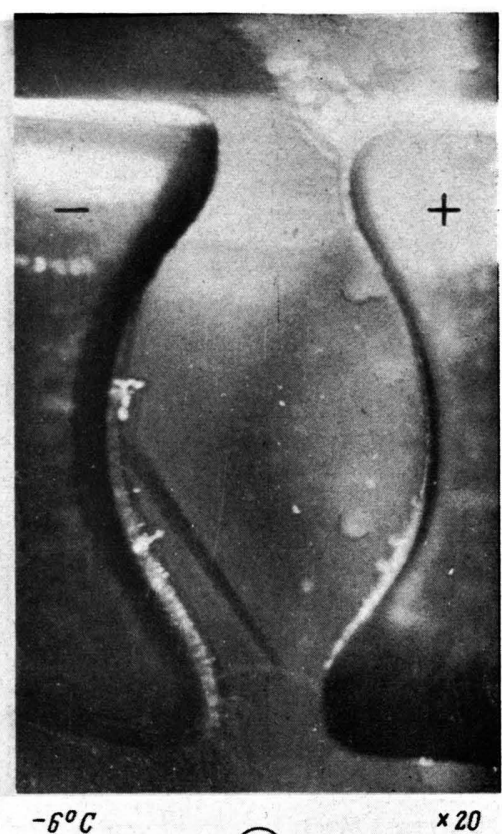

(17)

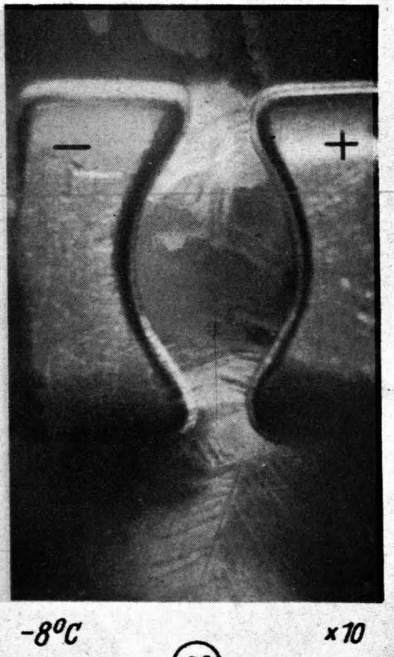

(20)

Aufnahmen 15-20. 
schon die Büschelentladung Eisbildung, hervorrufen kann, besagt, daß hier offenbar schon geringere Feldstärken zu einer ausreichenden dielektrischen Polarisation führen können. Dies wäre am einfachsten durch gewisse Strukturänderungen ${ }^{8}$ im Wasser zu verstehen, die evtl. auch für das Zustandekommen des zweiten Maximums des Gefrierkernspektrums verantwortlich sind. Dieser Temperaturbereich ist mehrfach ausgezeichnet. Auch der Dampfdruckunterschied zwischen Wasser und Eis ist hier am größten und die Kristalltracht ändert sich.

Alle bei der Eisbildung durch elektrische Funken beobachteten Erscheinungen können am einfachsten durch die dielektrische Polarisation in dem ihn veranlassenden elektrischen Feld erklärt werden. Es ist aber zu bedenken, daß der elektrische Funke selbst ein so komplexes Ereignis darstellt, daß es schwer fällt, alle seine Nebenerscheinungen von der Eisbildung sauber auszuschließen. Da, wie gesagt, schon stark abgetrocknete Kerne zur Eisbildung ausreichen, und keine Isomorphie mit Eis notwendig ist, könnte die Keimbildung bereits dadurch hervorgerufen werden, daß beim Funken Teile der Elektrode (Platin, Kohle, Messing) mitgerissen werden bzw. die starke Erwärmung zur örtlichen Abtrocknung von Kernen führen könnte. Auch die sonst unwirksame Erschütterung der Tropfen könnte beim Funkenüberschlag schließlich doch eine besondere lokale Heftigkeit erreichen und keimbildend werden. Selbst die beobachtete Orientierung der Eisgebilde ist nicht ganz eindeutig. Sie könnte u. U. nur das Ergebnis einer ausrichtenden Wirkung des elektrischen Feldes auf Eisgebilde sein, die auf ganz andere Art und Weise entstanden sind.

Es galt daher, alle diese Nebenerscheinungen auszuschließen. Sollten beim Funken tatsächlich Gefrierkerne entstehen, so müßten sie sich vielleicht durch eine Ölschicht auf den Tropfen abfangen lassen. Während viele Beobachtungen dafür vorliegen, daß eine Ölschicht auf Wasserproben in Glaskapillaren und offenen Gefäßen die Unterkühlung begünstigt, was wohl nur daher kommen kann, daß dann die Gefrierkerne nicht mehr aus dem Luftraum ins Wasser eindringen können, gelang die elektrische Gefrierauslösung in Wassertropfen mit einer Ölschicht schon

8 Vgl. die Änderung der Zusammensetzung des Wassers aus einfachen Molekeln und Assoziaten von zwei, vier und acht Molekeln mit der Temperatur nach A. E u c k e n, Z. Elektrochem. angew. physik. Chem. 53, 102 [1949].

9 L. Dufour, Poggendorf Ann. Physik 114, 530 [1861]. um $-4^{\circ} \mathrm{C}$, und zwar auch in verunreinigtem Wasser, wo sie sonst erst bei $-10^{\circ} \mathrm{C}$ möglich war. Das deckt sich mit den Ergebnissen von D u f o u r ${ }^{9}$, der ohne Angabe einer Temperaturgrenze nur berichtet, daß die Eisbildung in seinen in Öl schwebenden Wassertropfen beim Funken immer eintrat und die Erschütterung der Tropfen beim Funken dafür verantwortlich machte. Daß das Wasser durch die Beigabe von Öl reiner wurde, ist nicht anzunehmen. Zudem war der Effekt auch zu erzielen, wenn man Metallpulver auf die Tropfen aufstreute; wieder rückte dadurch die elektrische Eisbildung von $-10^{\circ} \mathrm{C}$ herauf auf $-4^{\circ} \mathrm{C}$. Diese Erscheinung ist in Kurve $4 \mathrm{a}, \mathrm{Abb} .1$, in der spektralen Verteilung eingetragen. Der Grund dafür muß in einer Änderung des Feldaufbaus liegen; denn jetzt treten auch beim verunreinigten Wasser die langen Gleitfunken auf. Die Struktur der Eisgebilde ist mit der bei reinem Wasser identisch; es können also auch beim Funken nicht einfach durch Oxydation des Öls oder Metalls frische Gefrierkerne gebildet worden sein, denn solche müßten in diesem Temperaturgebiet immer Nadelgebilde und keine Sterne verursachen.

b) Elektrische Eisbildung durch hohe Felder ohne Funken

\section{a) Eiskeimbildung an Spitzen}

Um von den Nebenerscheinungen des elektrischen Funkens loszukommen, gleichzeitig aber genügend hohe Feldstärken im Tropfeninnern zu erhalten, wurde die Spitze direkt in den Wassertropfen getaucht. Es kommt dann aber einfach wieder zur Entladung, und zwar als kurzes Büschel in der Oberfläche. Um dies $\mathrm{zu}$ verhindern, muß der in der Tropfenoberfläche liegende Querschnitt der Elektrode groß sein. Dazu wurde eine Kugel von $3 \mathrm{~mm}$ Durchmesser an einem Metallstäbchen sitzend etwa zur Hälfte in den Tropfen eingetaucht; der eingetauchte Teil trug eine kurze, möglichst feine Spitze. Diese Anordnung war sehr günstig: in mehrfach destilliertem Wasser kam es schon $\mathrm{ab}-3^{\circ} \mathrm{C}$ und schon mit Spannungen von $0,5 \mathrm{kV}$ zur Eiskeimbildung. Dabei erwies es sich als zweckmäßig, wenn man die Spannung nicht lange angelegt ließ, sondern nur eine Kondensatorentladung $(1 \mu \mathrm{F})$ auf den Tropfen gab ${ }^{10}$.

Diese Eisbildung spricht ganz besonders für die dielektrische Polarisation. Bei höheren Spannungen (4 bis $7 \mathrm{kV})$ und geringerer Unterkühlung $(-3$ bis $-5^{\circ} \mathrm{C}$ ) wird im Augenblick des Einschaltens der Hochspannung ein ganzer Strahl kleiner Eissternchen 
von der Spitze weg in den Tropfen hineingetrieben; bei niedrigeren Spannungen ( 1 bis $2 \mathrm{kV}$ ) bleiben die Sterne auch an der Spitze (Aufnahme 10) oder es wächst überhaupt nur ein einziger Stern an der Spitze (Aufnahme 11 und 12) so orientiert, daß eine Diagonale (Nebenachse) in Spitzenrichtung verläuft, also in der Richtung größter Feldstärke. Bei stärkerer Unterkühlung ergibt sich das mehr flächenhafte Auswachsen der Eisgebilde von der Spitze aus durch den ganzen Tropfen hindurch.

\section{B) Eisbildung an kleinen Kugeln; die notwendigen Feldstärken}

Da bei unserer Versuchsanordnung die erforderliche Reinheit des Wassers noch nicht genau genug gewährleistet war und auch die wegen der Wassertropfen nur klein dimensionierten Felder nicht homogen genug sein dürften, kann die notwendige Feldstärke aus den bisherigen Beobachtungen nur geschätzt werden. Das gilt vor allem beim Funken, bei dem in der Luft eben die Durchbruchsfeldstärke erreicht wird, der im Wasser wirksame Anteil aber undefiniert ist.

Etwas genauere Werte ergaben Versuche mit Metallkugeln von verschiedenem Durchmesser, die sich möglichst weit von der Unterlage entfernt in der Tropfenkuppe befanden. Damit keine Wirkung der Zuleitung zur Kugel störend hereinkommen konnte, wurde diese ganz mit Glas verkleidet. Bei solchen Kugelelektroden (ohne Spitze) verläuft die Eisbildung immer gleichmäßig radial von der Kugel aus nach außen, also wieder in der Richtung der Feldlinien. Zum Teil werden bei zu hoher Spannung auch hier Eissternchen nach außen getrieben.

10 Nach J. M a ls ch, Physik. Z. 30, 837 [1929], wird auch bei der Messung der Dielektrizitätskonstanten in hohen elektrischen Feldern nur eine Kondensatorentladung benützt, sobald es sich um Flüssigkeiten mit hohen Dielektrizitätskonstanten handelt. Die hohe Spannung ist dann nur während einer sehr kurzen Zeit $\left(10^{-7}\right.$ bis 10-8 Sek.) angelegt; die Flüssigkeiten mit großen Dielektrizitätskonstanten haben im allgemeinen eine so große Leitfähigkeit, daß beim längeren Einschalten einer höheren Spannung Erwärmungen auftreten. Diese sind auch bei unseren Versuchen unerwünscht; vermutlich werden dann auch keine genügenden Feldstärken erreicht.

Die Messungen von $\mathrm{Mals \textrm {ch }}$ zeigen übrigens, daß zur Erreichung des Sättigungszustandes, bei dem alle Dipole vollkommen gerichtet sind, Feldstärken notwendig wären, die weit über den Durchbruchsfeldstärken liegen. Der Beginn der elektrischen Sättigung ist aber schon bei Feldstärken von etwa $30 \mathrm{kV} / \mathrm{cm} \mathrm{zu}$ beobachten. (Siehe A. E u c k e n, Lehrb. der chem. Physik, II, 2. Teil, 2. Aufl., S. 864:)

\begin{tabular}{|c|c|c|c|c|}
\hline \multicolumn{5}{|l|}{ Elektrode } \\
\hline Art & 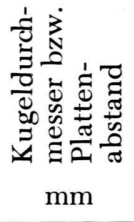 & 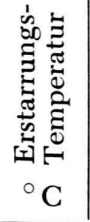 & 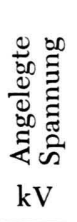 & 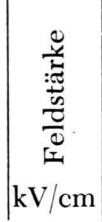 \\
\hline $\begin{array}{l}\text { Pt-Kugel } \\
\text { geschmolzen }\end{array}$ & 0,5 & $\begin{array}{l}-4 \\
-6\end{array}$ & $\begin{array}{l}1 \\
0,5\end{array}$ & $\begin{array}{l}40 \\
20\end{array}$ \\
\hline $\begin{array}{l}\text { Pt-Kugel } \\
\text { geschmolzen }\end{array}$ & 1 & $\begin{array}{l}-4 \\
-6\end{array}$ & $\begin{array}{l}3 \\
1,5\end{array}$ & $\begin{array}{l}60 \\
30\end{array}$ \\
\hline $\begin{array}{l}\text { Neusilber-Kugel } \\
\text { gedreht }\end{array}$ & 2 & -7 & 2 & 20 \\
\hline Kondensator, Aufn. 15 & 1,0 & -7 & 2 & 20 \\
\hline Kondensator, Aufn. 16 & 1,0 & -6 & 2 & 20 \\
\hline Kondensator, Aufn. 17 & 0,8 & -6 & 1,5 & 19 \\
\hline Kondensator, Aufn. 18 & 0,7 & -7 & 1,5 & 22 \\
\hline Kondensator, Aufn. 19 & 0,7 & -7 & 1,5 & 22 \\
\hline Kondensator, Aufn. 20 & 0,7 & -8 & 1,5 & 22 \\
\hline
\end{tabular}

Tab. 1. Zur Eisbildung benötigte Feldstärken.

In Tab. 1 sind die erforderlichen Mindestfeldstärken zusammengestellt. Außer den Werten für die Kugelelektroden enthält sie noch Ergebnisse aus Versuchen mit Plattenkondensatoren, die in Abs. II, $2 \mathrm{c}$ genauer beschrieben sind. Trotz der den Werten anhaftenden Unsicherheiten sieht man doch, daß im Durchschnitt etwa $20 \mathrm{kV} / \mathrm{cm}$ bei Unterkühlungen auf -6 bis $-7^{\circ} \mathrm{C}$ benötigt werden; bei $-4^{\circ} \mathrm{C}$ ergeben die beiden vergleichbaren $\mathrm{Pt}-\mathrm{Kugeln}$ einen Wert von etwa $50 \mathrm{kV} / \mathrm{cm}$. An Spitzen wären die errechenbaren Feldstärken noch höher; sicher werden aber gerade diese hohen Felder nicht erreicht. In Tab. 1 kommt die bei den Versuchen auch sonst zu beobachtende Abhängigkeit der Feldstärke von der Unterkühlung zum Ausdruck. Bei stärkerer Unterkühlung, also geringerer Eigenbewegung der Moleküle, genügen schon schwächere Felder. Bemerkenswert ist, daß nach den Versuchen von $\mathrm{Malsch}{ }^{10}$ geschlossen werden kann, daß von $30 \mathrm{kV} / \mathrm{cm}$ ab eine so große Anzahl von Dipolen im Wasser gleichgerichtet ist, daß schon der Beginn der elektrischen Sättigung zu beobachten ist.

\section{r) Eisbildung an Elektroden mit Bernsteinhülle}

Solange bei den Versuchen die Metallelektroden direkt mit den Wassertropfen in Berührung kommen, sind immer noch Einwände dagegen denkbar, daß die Eiskeimbildung nur durch die dielektrische Polari- 
sation verursacht wird. Auch hier können beim Anlegen der Hochspannung noch Metallionen aus der Elektrode austreten. Aber Ionen allein sind nicht wirksam; sie treten auch bei niedrigen Spannungen aus, wo die Versuche jedoch keine Eisbildung ergaben. Auch starke Ionisation der Luft in unmittelbarer Nähe der Tropfen durç ein dicht neben dem Tropfen liegendes radioaktives Präparat blieb ohne Wirkung, was nicht weiter verwundert, denn Ionen ständen ja schon immer genügend aus Ultrastrahlung und radioaktiven Verunreinigungen im Laboratorium und in der Atmosphäre zur Verfügung, und ihre Wirkung wäre längst bemerkt worden.

Die reinsten Verhältnisse hat man, wenn man das Wasser nicht mit dem Metall der Elektrode in Berührung kommen läßt, indem man die Metallelektrode mit Bernstein umkleidet, so dick natürlich, daß kein Durchschlag erfolgt. Die Spannung ist dabei zu erhöhen; bei 4 bis $7 \mathrm{kV}$ tritt im Augenblick des Anlegens der Spannung sofort vom Bernstein aus die Eisbildung in der für diesen Temperaturbereich üblichen Struktur ein (Aufnahme 13). Dabei kann es sich nur um dielektrische Polarisation handeln.

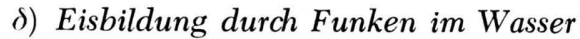

Die geringste Unterkühlung war notwendig, wenn die Eisbildung durch kleine Funken veranlaßt wurde, die zwischen zwei im Innern des Wassertropfens befindlichen Spitzen mit ganz geringer Entfernung übersprangen. Solche Funken wirkten schon ab $-1^{\circ} \mathrm{C}$ und das auch in leicht verunreinigtem Wasser. In diesem Fall bildet sich das elektrische Feld im Tropfeninnern, die abschirmende Wirkung der Oberfläche fällt weg und man kann mit verhältnismäßig hohen Feldstärken rechnen. Auch diesmal wird eine Unmenge kleiner Eissterne und Plättchen gebildet (Aufnahme 14).

Diese Eisbildung durch Unterwasserfunken ist deshalb besonders wichtig, weil man daraus ersieht, daß der Temperaturbereich des ersten Maximums des Gefrierkernspektrums keineswegs die obere Grenze der Unterkühlungen darstellt, bei denen die elektrische Gefrierauslösung gerade noch möglich ist. Diese liegt, wie bei der Impfung mit frischen Kernen, noch viel näher bei $0^{\circ} \mathrm{C}$, und das Maximum bei $-4^{\circ} \mathrm{C}$ kommt erst wieder durch Nebenwirkungen, wie Reinheit des Wassers, Erwärmung durch Funken und Variation gewisser Versuchsbedingungen im größeren Mittel, zustande.
ع) Zusammenstellung der verschiedenen Arten der elektrischen Gefrierauslösung

Die Untersuchungen haben ergeben, daß die elektrische Eiskeimbildung bei genügend tiefen Temperaturen immer eintritt. Die Wirkungsbereiche der Elektrodenanordnungen liegen etwas verschieden und können durch die entsprechenden Häufigkeits-

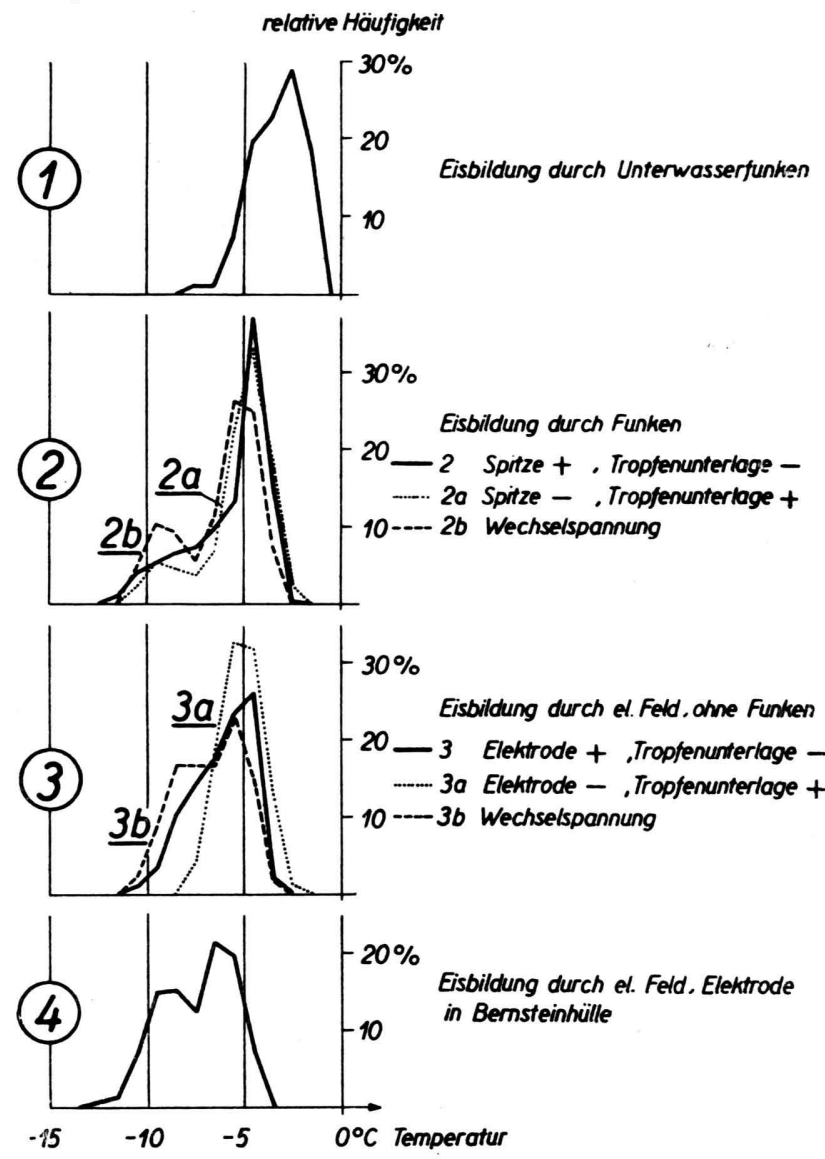

Abb. 2. Elektrische Gefrierauslösung bei verschiedenen Elektrodenanordnungen.

verteilungen der mit ihnen erzielten Gefriertemperaturen (Abb. 2) miteinander verglichen werden.

Die beste Wirkung hat der Unterwasserfunke; er wirkt schon von $-1^{\circ} \mathrm{C}$ ab (Kurve 1); Funken im Luftraum (Kurve 2) über den Tropfen hinweg und Anlegen der Hochspannung an Elektroden im Tropfeninnern (Kurve 3) wirken im Mittel gleich gut. Dabei ist die Elektrode positiv, die Tropfenunterlage negativ und geerdet. Umpolen (Kurve 2 a und 3a) gibt keinen merklichen Unterschied. Dasselbe gilt für die Kurven $2 \mathrm{~b}$ und $3 \mathrm{~b}$ bei Verwendung von 
Wechselspannung. Am schlechtesten ist die Wirkung bei mit Bernstein umhüllten Elektroden (Kurve 4). Die Unterschiede zeigen deutlich den Einfluß der Feldstärke, die beim Unterwasserfunken am höchsten, bei Bernsteinhüllen am schwächsten sein dürfte. Die zu jeder Verteilungskurve führenden Versuchsserien wurden mit einer großen Anzahl immer wieder frisch destillierter Wasserproben ausgeführt, so daß der Reinheitsgrad im Mittel gleich ist. Auch die Variationen der Feldstärke bzw. angelegten Spannung sind für alle Kurven im Mittel etwa dieselben. Die Kurven sind also wirklich miteinander vergleichbar.

c) Die Orientierung der entstehenden Eisgebilde im elektrischen Feld

Bei den bis jetzt beschriebenen Versuchsanordnungen erwies sich die elektrische Eisbildung von der Polung unabhängig, und die entstehenden Eissternchen und -Kreisscheibchen standen immer mit einer Flächendiagonale in der Richtung der Feldlinien. Es ist aber zu bedenken, daß diese Richtungen mit Ausnahme des Funkens auch durch das größte Temperaturgefälle in den Tropfen ausgezeichnet sind, sobald an der Spitze oder an der Kugel ein Eiskeim entsteht, was zur Folge haben könnte, daß der Keim in diesen Richtungen am schnellsten wachsen und dadurch in manchen Fällen auch eine gewisse Orientierung der Eiskristalle zustandekommen würde. Die Entscheidung war durch die Wahl geeigneter Feldformen zugunsten der dielektrischen Polarisation herbeizuführen.

Bringt man einen kleinen Plattenkondensator in einen Wassertropfen - der Tropfen liegt dabei auf einer Plexiglasplatte in einer ihn fassenden Mulde und die Platten müssen zur Vermeidung von Spitzenwirkung als kleine Klötzchen ausgebildet sein -, so verlaufen die im Augenblick des Anlegens der Spannung entstehenden Eisgebilde immer in ganz bestimmten Richtungen von den Platten weg nach außen (Aufnahme 15), wobei Richtungen von $60^{\circ}$ gegen die Kondensatorachse bevorzugt sind. Ohne elektrisches Feld gefriert derselbe Tropfen bei derselben Versuchsanordnung ohne jegliche Orientierung und $\mathrm{zu}$ einem dem Zufall überlassenen Zeitpunkt. Besonders interessant ist die Anordnung der Eisteilchen im Innern des Kondensators (Aufnahme 16). An den ebenen Plattenstücken verlaufen die Eisfedern senkrecht von einer Platte zur anderen hinüber und wachsen unter $60^{\circ}$ dazu aus; im oberen
Teil des Kondensators ist das Wachstum mehr räumlich und die Orientierung nicht so klar. Daß hier eine Hohlelektrode verwendet wurde, hat seinen Grund darin, daß die elektrischen Feldlinien immer senkrecht zur Leiteroberfläche austreten und damit an der konkaven Fläche eine Fokussierung der Eisteilchen erwarten lassen. Dieses radial nach innen gerichtete Ansitzen der ersten Eisteilchen konnte in Aufnahme 17, linke Platte, gerade festgehalten werden. Mitunter kommt es sogar vor, daß sich die Eisnadeln im Krümmungsmittelpunkt der Hohlelektrode überschneiden. Bei geringen Unterkühlungen handelt es sich bei diesen ersten Eisgebilden immer um ganz dünne, runde Eisscheibchen oder -sternchen, die wieder mit ihrer größten Erstreckung (Nebenachse) senkrecht zur Elektrode, also in Richtung der Kraftlinien sitzen. Das kann man in den Aufnahmen 18 und 19 deutlich sehen, wo sie zudem an der engsten Stelle des Kondensators und damit im Gebiet größter Feldstärke entstanden sind. Die hellen Plättchen in Aufnahme 18 haben sich soeben abgelöst und gedreht, so daß sie im reflektierten Licht genau, aber nicht mehr in der ursprünglichen Lage zu sehen sir 1 . Beide Aufnahmen zeigen außerdem ganz deutlich, daß eine sehr große Anzahl von Eiskeimen gleichzeitig entsteht; diese wachsen gar nicht alle aus und das endgültige Durcherstarren der Tropfen geschieht in ganz anderen, durch die Temperaturverhältnisse bedingten Richtungen. Diese Orientierung der Eisteilchen spricht also auch eindeutig für die dielektrische Polarisation.

Ein Einfluß der Polung ist an sich nicht zu erwarten und konnte in unseren Versuchen auch nicht eindeutig erfaßt werden. Zwar hatte die negative Platte meistens den Eisteilchenansatz, und das auch, wenn der Kondensator zur Eliminierung von Feldinhomogenitäten umgepolt wurde. In vielen Fällen traten die Eisteilchen aber auch an der positiven Platte auf. Und Aufnahme 20 gibt einen Fall wieder, wo die radial ansitzenden Eisteilchen auf beiden Platten zugleich vorkommen. Vielleicht entscheiden hier auch kleine Inhomogenitäten des Feldes, die bei der Kleinheit der hier verwendbaren Elektroden schon durch deren Kanten und Unebenheiten verursacht werden können.

\section{Schlußfolgerungen}

Die beschriebenen Beobachtungsergebnisse lassen zưsammen erkennen, daß ein genügend hohes elektrisches Feld die Eiskeimbildung bei entsprechender Unterkühlung immer hervorruft und daß man diese Wirkung der dielektrischen Polarisation des Wassers 
im elektrischen Feld zuschreiben kann. Damit ist ein neuer Zusammenhang zwischen Elektrizität und Eisbildung gefunden, welcher in gewissem Sinne den Umkehreffekt zu der schon lange vermuteten elektrischen Tönung der Eisbildung darstellt, die neuerdings von Workman und Reynolds ${ }^{11}$ an in Wasser wachsendem Eis nachgewiesen werden konnte.

Der Abtrocknungseffekt der Gefrierkerne und die gute Impfwirkung von trockenen Kernen bei unterkühlten Tropfen können also recht gut durch die Wirkung einer elektrischen Doppelschicht zustande kommen. Im übrigen muß die elektrische Eisbildung überall dort vorkommen, wo genügend hohe Feldstärken zur Verfügung stehen, vor allem also bei Gewittern, wo es dadurch spontan zur Eisbildung und infolge der freiwerdenden Erstarrungswärme zu neuem Auftrieb der aufsteigenden Luftmassen kommen muß. Gefrierkerne sind dazu nicht notwendig.

Vorläufige Untersuchungen an Regenwasser ergaben übrigens, daß das Wasser aus starken Regenfällen im Sinne dieser Arbeit als sehr rein zu bezeichnen ist; die elektrische Eisbildung gelingt dementsprechend bei geringen Unterkühlungen. Wasser aus schwachen Regenfällen oder aus dem Beginn von Regenfällen hatte mehr die Merkmale des verunreinigten Wassers.

Eine neue Wirkung müßte auch beim Lenard-Effekt auftreten. Er besteht bekanntlich darin, daß aus Regentropfen durch einen Luftstrom von stark

11 E. J. Workman u. S. E. Reynolds, Physic. Rev. 74, 709 [1948]. schwankender Heftigkeit kleine Tröpfchen $\left(10^{-6} \mathrm{~cm}\right)$ mit negativer Ladung aus der Oberflächenschicht nach oben mitgerissen werden, während größere positiv geladene Tropfenreste absinken, wobei das starke Ausziehen der Kraftlinien zu sehr hohen Spannungen, ja vielleicht zur Gewitterelektrizität führen kann. Nach unseren Beobachtungen müßte es dabei zur Eisbildung kommen, sobald es sich um unterkühltes Wasser handelt, das im allgemeinen in der Atmosphäre auch oberhalb der $0^{\circ}$-Isotherme noch vorherrscht ${ }^{2}$. Beim Zerblasen der ursprünglichen Doppelschicht werden neue Doppelschichten gebildet, und die Ausrichtung der Wassermoleküle muß elektrische Eisbildung geben. Vorläufige Untersuchungen, wobei mit einem Luftstrahl aus einer feinen Düse in einen unterkühlten Tropfen geblasen wurde, ergaben die von der elektrischen Eisbildung her bekannte Erscheinung: sofort beim Einblasen entstehen schon bei Temperaturen von $-1^{\circ} \mathrm{C}$ unzählige Sternchen und Eisplättchen (wie in Aufnahme 4) in einem einzigen Wassertropfen. Nach der Coehn schen Regel müssen sich diese Eisteilchen im Wasser wegen der kleineren Dielektrizitätskonstante des Eises negativ gegenüber den Wasserresten und damit in dem zu den neueren Gewitterbeobachtungen passenden Sinn aufladen, falls nicht der bekannte Einfluß von Ionenbeimengungen bei der Polung den Ausschlag gibt. Da bei der Deutung von Eiskeimbildungen, wie im Verlaufe dieser Arbeit mehrfach zum Ausdruck kam, größte Vorsicht geboten ist, müssen hier die Entstehungsbedingungen noch genauer untersucht werden. 